\title{
Likeness and Dealbreakers: Interpreting Interpersonal Compatibility from Online Music Profiles
}

\author{
Mo Kudeki and Karrie Karahalios \\ University of Illinois \\ kudeki@uiuc.edu, kkarahal@cs.uiuc.edu
}

\begin{abstract}
How much would a stranger know about you if they knew only the last song you listened to? Quite a bit, at least according to the stranger. Music taste often acts as proxy for our personalities, and we constantly perform this taste perception online. In our study, we find that good first impressions are common, bad first impressions are extreme, and that people pick up on many subtle cues about personality and whether they share common values based on data found in an online music profile. Similar motivations for listening to music and the perception of individuality are more highly valued than similar taste in genres or artists, suggesting that social media applications could benefit from incorporating these motivations in predicting compatibility between users.
\end{abstract}

Keywords: Music, listening history, taste performance, impression formation, personality, social networking service.

\section{Introduction}

Describing one's music taste is typically a difficult task. However, we do so indirectly every day on social networking profiles, either by explicitly listing favorite bands, or simply by listening to music on a computer or mobile device and having its song data automatically uploaded ("scrobbled") to social network sites such as Last.fm.

Up-to-the-minute information about what your friends are listening to is available on Facebook, or within music listening applications such as Spotify and Rdio. As music listening habits and histories become more public, people have the almost constant opportunity to pass judgments - good or bad - about their friends' and acquaintances' music taste.

People feel that knowing someone's music taste provides insight into them as a person [11]. Likewise, users of Last.fm feel that their music taste "signifies aspects of their identity that their community of peers may read," [2]. It is not surprising then that careful impression management and profile work is pervasive on Last.fm; people deliberately manipulate their own profiles, deleting embarrassing songs, even going so far as to queue up a list of music, hit play, and then leave the room [10]. The purpose of this curation is to communicate to their peers on social networks a particular statement, often of prestige/expertise, association with a particular subculture, or to differentiate oneself from their friends [4]. 
Perhaps surprisingly, stereotypes about fans of different musical genres are quite accurate, down to predicting specific personality traits and drug preferences [7]. Listening to a CD of someone's favorite songs even provides a more accurate depiction of their personality than other zero-acquaintance methods [8].

There are many studies about impression management on social networks, and about music-based stereotypes (offline). People care about how others perceive their musical taste, and put significant time and effort into making sure their profiles signal the right impression. Less is known about how people interpret these curated music profiles online. This is where we focus our study.

\section{Methods}

Our goal in this study was to uncover how people understand each other's music taste and personalities based on the limited music preference information available on a social networking site profile.

We chose Last.fm as the social media platform for our study for several reasons. First, the central focus of Last.fm is music taste and music discovery, so its users are familiar with viewing the music listening histories of other users. Second, Last.fm maintains a long listening history (it was founded in 2003) and collects a large amount of data about each user (every music listen scrobbled from a user's iTunes library, for example); we could easily collect months or years of listening history from actual users. Third, Last.fm's API and open privacy policy make user listening data publicly accessible [6].

We created an online survey where subjects viewed and rated others' Last.fm profiles, in addition to answering a few questions about their own music-listening habits. We then showed the subject a series of five profiles filled with other, anonymous Last.fm users' listening histories. The data visible to the subject rating a particular profile is that person's: last 15 song listens, top artists over the past 3 months with playcounts, and top artists overall with playcounts.
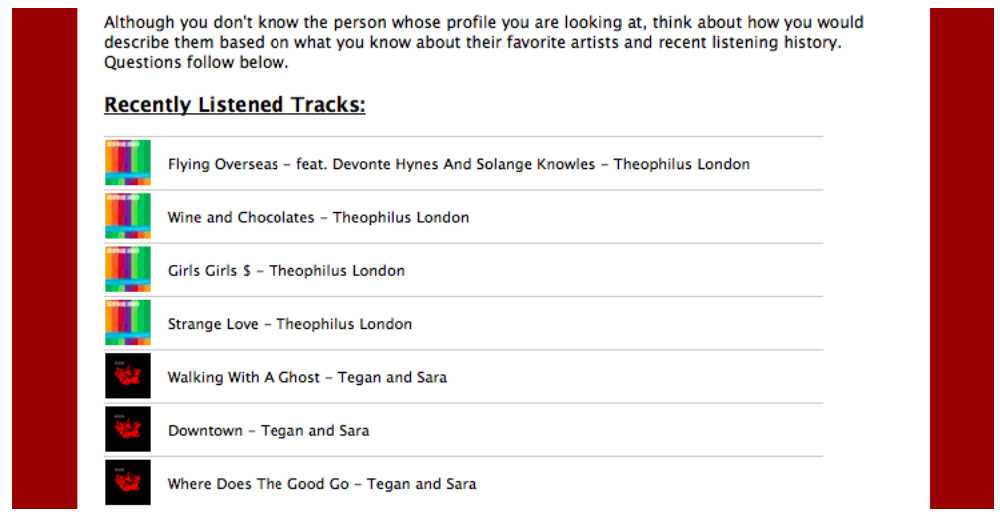

Fig. 1. Sample profile view in the survey (recent listens) 


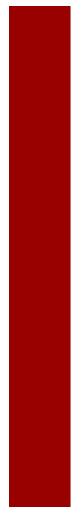

Top Artists for the past 3 months:
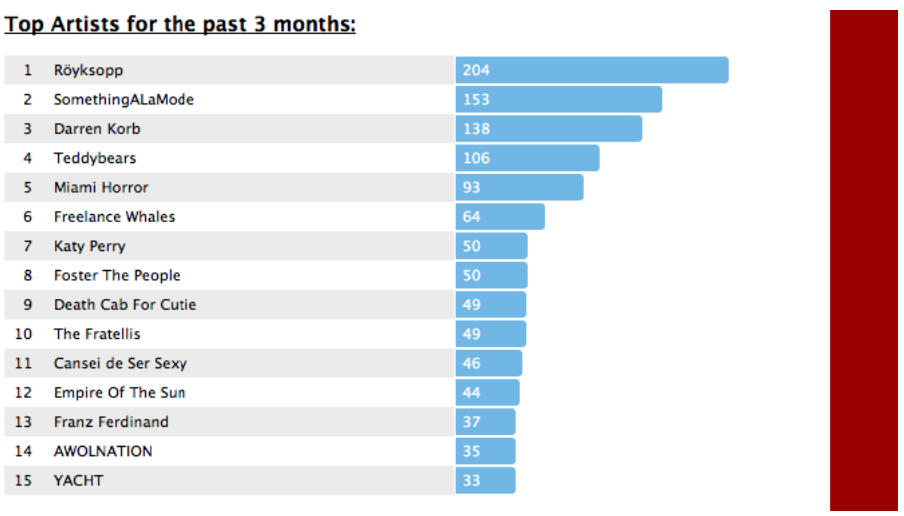

Fig. 2. Sample profile view in the survey (top artists)

We chose to use the last 15 song listens because it gave the subject a slice-of-time view of actual listening history - what songs are listened to together in one sitting, while the other two sections contain aggregate data. The intention was to provide insight to a profile owner's current taste (the 3-month window) alongside their longterm favorite artists. If the profile owner has only used Last.fm for a short time or has very constant taste, these two lists will be similar, whereas users with a longer account history or rapidly changing taste may have little to no overlap.

For each of the five profiles, the subject filled out a questionnaire where they described their perceived music taste and personality traits of the profile owner. Most questions were open-ended in short-answer format. Subjects did not have access to certain information that is typically present on a real Last.fm profile page, such as an avatar, username, age, location, and gender. We wanted to see what guesses subjects made about demographic information based solely on music listening history. Upon completion of the survey, the subject's own Last.fm profile information (the three sections listed above) was stored and rated later by four other study participants.

\subsection{Design of Study Interface}

Participants logged into the survey by authenticating with their Last.fm accounts. They first answered a few questions about themselves: their age, occupation, how often they log into Last.fm, and how many hours per day they spend listening to music. The subject then proceeded to rate and answer questions about each profile.

We made the design decision to show only a very limited amount of music listening history (up to 30 different artists and 10 song/artist pairs) in order to expose the subject to roughly the same amount of information one can glean by glancing at a real Last.fm profile. We limited the information presented to help simulate forming an immediate first impression as one would on Last.fm.

The survey interface (Figures 1-2) is designed to look as much like an actual Last.fm page as possible. The profile information is displayed using the same layout, styling, and images used on the Last.fm site, down to the rendering of the playcount bars' lengths. We wanted subjects to have a familiar browsing experience. 


\subsection{Survey Question Content}

The questions about the profiles subjects rated focused on two main areas: the profile owner's music taste (three questions), and how they perceive the profile owner's personality, demographics, lifestyle, and whether they think they would get along with the profile owner (three more questions). Given the small number of artists present in a profile, it may be more difficult to make statements about a profile owner whose artists were unfamiliar. To provide context for this, we asked, "How many of the artists in this user's profile are you familiar with?"

\subsection{Implementation}

We built the survey using PHP with MySQL and handled the Last.fm API calls through a third-party library, PHP Last.fm API [5]. When a subject logged into the survey with their Last.fm credentials, their profile was checked to verify they had enough data/activity to qualify as a sample profile for future survey participants.

The survey requested that the subject rate the anonymous profiles of the five most recent survey participants. This assignment strategy wouldn't work unless there were already five profiles in the database, so seed profiles (selected by browsing Last.fm) were used at the beginning of the study. Subjects' responses about these profiles were discarded. When the survey was completed, we stored the five answer sets, as well as the current subject's listening history to show to future participants. We discarded the identifying username, making all the responses anonymous.

The survey ran for approximately one week. Advertising was done entirely online, on Last.fm forum pages, the Facebook page and SoundCloud group for Last.fm users, on Tastebuds.fm forums (a Last.fm-based dating website), and via email and Facebook amongst student groups at a large university. A total of 48 participants completed 225 profile ratings.

\subsection{Response Coding}

We used mixed methods to analyze our responses. We counted word frequencies for the listed genres and adjectives used to describe profile owners, and took averages of the questions with numerical responses (recognized artists, time per day spent listening to music). We qualitatively examined all 225 responses and performed an open-ended coding on the short-answer questions, sorting recurring ideas and patterns into categories. We focused specifically on comments about the profile owner's personality and presumed compatibility with the subject. The coding approach we used was similar to a University of Kansas study exploring stereotypes of Chinese international students, compiling an open-ended list of descriptors into a collection of stereotypes. This study used these participant-generated stereotypes as content for a later part of the study [9]. The common genres, stereotypes, and descriptors we found could be used in a follow-up study investigating the accuracy of perceived personality traits based on music profiles. 


\section{$3 \quad$ Results}

Participants ranged in age from 18-55, averaging 23.6 years old, and around $40 \%$ were students. They were avid music listeners and active Last.fm users, self-reporting an average of 4.85 hours spent listening to music per day. 40 out of 48 log into Last.fm multiple times per month ${ }^{1}$. Musical recognition of bands in others' profiles was high, with subjects recognizing at least 10 artists in a particular profile more than $60 \%$ of the time.

The four most common genres listed were "rock", "indie", "pop", and "electronic", which accounted for 117 out of 374 total genre mentions. However, out of 120 unique genres mentioned, 69 of them are only mentioned a single time, meaning just once by a single subject. Many of these genres were clearly chosen to sound either very unique ("dadrock", "library ghetto"), or to fill a gap where traditional genre names lacked sufficient nuance ("foreign synthpop", "post-rock ambienty"). If we count combination genres like these that include at least one of the top four genres (e.g. "indie-folk", "electronic ambient"), this accounts for roughly another third of genre mentions (103 out of 374). The wide range of these genre names suggests that participants may have been trying to display their own musical expertise and individuality in their evaluations of others.

Responses about the profile owners clustered into two major themes: whether or not the subject thought they would get along with the profile owner (compatibility), and a more holistic analysis of the profile owner's life experience, personality, and psyche, based on cues in the listening data.

Could We Be Friends? Subjects believed they would get along with an arbitrary profile owner $53 \%$ of the time, thought they wouldn't $32 \%$ of the time, and had a more lukewarm response ("maybe" or "unsure") just $15 \%$ of the time according to our coding. However, they tended to rate others' music taste as more different than similar to their own (an average rating of 2.41 out of 5). Subjects thought liking the same bands would give them something to talk about, or that they could relate to someone with similar taste to their friends:

- "Most likely, at the very least we can bond over Muse."

- "Yep. Sigur Ros, Andrew Bird and Radiohead? The bands I don't know sound pretty fun too."

- "Yes. I know a few people that enjoy this genre; they're pretty wonderful."

Some subjects believed that even if a friendship were unlikely, they may get along as casual friends and thought they could identify what drugs they could do together:

- "They seem outgoing, bubbly, easy to converse with. They might move on before anything gets meaningful, though."

- "I do like old school rap and can maybe get high with him"

${ }^{1}$ Our sample may have been self-selecting, since the study was advertised as a survey about music taste. Our subjects were passionate about music, but this may not be representative of all Last.fm users. 
The tendency to give others the benefit of the doubt is found often in Computer Mediated Communication studies. People rate others higher when fewer cues are present, assuming the person in question has similar attitudes to their own in the absence of more information [12]. However, even though the profiles in our study contain very few cues, certain "dealbreaker" bands evoke an intensely negative reaction. This happened in roughly $1 / 3$ of cases, and the subject typically deemed these "dealbreakers" so repulsive that a friendship would be impossible:

- "foster the people is unforgivable"

- "No. This guy likes Kanye West and Ani DiFranco. Not even Ratatat saves that."

- "He actually enjoys listening to James Blake whereas I just jump out the building whenever I hear his pretentious dickery."

Although negative impressions were less frequent, the character descriptions of these profiles were much stronger and harsher than the positive ones were positive:

- "This is a load of mopey almost-emo indie bullshit with a couple older (much more decent) bands thrown in to help this listener justify their continued existence."

- "this seems like the sort of person who gets their opinions from reddit and people like that are generally obnoxious."

Negative social stereotypes came up in these dealbreaker ratings, most frequently the "hipster" persona, often in conjunction with indie music. Comments often mentioned the gratuitous wearing of unnecessary apparel:

- "Guy wears glasses, maybe a scarf when not completely necessary"

- "Probably at college for film studies or some bullshit, works in the college coffee shop, likes taking shitty desaturated photographs on their DSLR camera and updating their microblog."

You Are What You Listen to. Cues in music profiles were used to make conclusions about a wide variety of traits, from organizational skills, to recent activities they'd done, to which relatives played an influential role on music taste during childhood:

- "College student who recently has been studying for finals. the reason for the classical music."

- "College age female whose parents had the common sense to introduce her to The Beatles before her identity was completely whitewashed."

One particularly important value to discern from a profile was whether or not this person valued seeking out new music for themselves. Participants in our study tended to be avid music listeners, and since music is an important part of their lives and identities, they reacted negatively when they saw profiles where that didn't seem to be the case. This was also assumed to be a shared value of members of the Last.fm community, and subjects appeared to prefer people who strive for individuality. They criticized profiles they saw as "mainstream" or only included the more well-known songs/artists of a particular genre: 
- "To this person, music is about being entertained, to me music is an art that is appreciated."

- "But seriously, a good indicator that I'll get along with someone is the amount of music they listen to that isn't on the billboard top 100. Nothing personal, but in general, liking lesser known music means one puts more thought into what they listen to, has a deeper taste other than 'catchy', and in general appreciates music more."

Similar motivations were more important than having bands in common, and lack of overlap can be an opportunity to learn from one another. Likewise, one could like the right band for the "wrong" reason:

- "Totally different music-listening taste - either this person would be my best friend after opening my mind up to new and wonderful things, or we will never get along because we'd both strongly believe the other should listen to our music."

- "I really like arcade fire, so I guess we like all of these things for different reasons? my aversion to muse may stem from how mainstream it is? although arcade fire is preeeetty mainstream at this point? (OH gosh I hate that word)"

Subjects were comfortable discerning specific personality traits from listening histories. Only a small minority (three responses) claimed that there would be no correlation between music taste and whether they would get along. The vast majority thought that personality came through loud and clear in the profiles:

- "Organized; they appear to prefer to listen to music by albums based on their recent tracks."

- "I don't like the bands I recognize, they sounds [sic] like a person from my same age group that I choose to not hang out with because there are too many cooler people out there"

- "Fun; musical diversity tends to be an indicator of this."

\section{Conclusion}

We found several identifiable trends about how people interpret each other based on music listening histories. People were bimodal about whether they could get along with someone, being either cautiously optimistic, or having an extremely negative reaction triggered by a single "dealbreaker" artist. Second, people detect behaviors, habits, motivations, and shared/unshared values from music profiles. These results give validity to the curation efforts people put towards their online music profiles. In particular, individuality and uniqueness were valued amongst our participants, warranting further study of whether this applies to a broader population.

Recent work [1] shows a number of factors that play into music listening decisions, and found, as we did, a difference "between conservative listeners and those more keen on experimenting." Another study [3] points out that what makes a mix tape good is the collection of songs chosen and the relationships between those songs. Yet there are few systems that recommend collections rather than individual items. In our 
study, we found that having shared artists was only one facet of music taste compatibility, and perhaps not the most important. Suggesting similar users is a common feature of social media, whether it be "neighbors" on Last.fm or matches on a dating website. We believe representing music taste as a collection of features (including dealbreakers, breadth of taste, attitudes towards finding new music, etc.) could help in suggesting better matches. We can also imagine using these findings in the reverse direction to build new music exploration interfaces to surface music enjoyed by like-minded people. The social traces we've found in this study could be leveraged to improve discoverability of both music and of people who are truly compatible, not just similar.

\section{References}

1. Baur, D., Büttgen, J., Butz, A.: A Large-Scale Principal Components Analysis of LongTerm Music Listening Histories. In: CHI 2012: Proceedings of the 2012 ACM Annual Conference on Human Factors in Computing Systems, pp. 1273-1276. ACM, New York (2012)

2. Fitzpatrick, C.: Scrobbling identity: Impression management on last. fm. Technomusicology: A Sand- Box Journal 1(2) (2008)

3. Hansen, D.L., Golbeck, J.: Mixing it up: recommending collections of items. In: CHI 2009: Proceedings of the 27th International Conference on Human Factors in Computing Systems, pp. 1217-1226. ACM, New York (2009)

4. Liu, H.: Social network profiles as taste performances. Journal of Computer-Mediated Communication 13, article 13 (2008)

5. Oakes, M.: PHP Last.fm API (updated September 6, 2011), https://github.com/matto1990/PHP-Last.fm-API/ (retrieved November 25, 2011)

6. Privacy Policy - Last.fm (February 11, 2009), http: / / www. last. fm/legal/privacy (retrieved December 17, 2011)

7. Rentfrow, P.J., Gosling, S.D.: The content and validity of music-genre stereotypes among college students. Psychology of Music 35, 306-325 (2007)

8. Rentfrow, P.J., Gosling, S.D.: Message in a Ballad: The role of music preferences in interpersonal perception. Psychological Science 17, 236-242 (2006)

9. Ruble, R.: Making Ourselves Understood: The Role of Previous Experience, Stereotypes, Communication Accommodation, and Anxiety in Americans' Perceptions of Communication with Chinese Students. Thesis, University of Kansas (2011)

10. Silfverberg, S., Liikkanen, L.A., Lampinen, A.: "I'll" press play, but I won't listen": profile work in a music-focused social network service. In: CSCW, pp. 207-216 (2011)

11. Voida, A., Grinter, R.E., Ducheneaut, N., Edwards, W.K., Newman, M.W.: Listening in: Practices surrounding iTunes music sharing. In: Proceedings of the ACM Conference on Human Factors in Computing Systems, CHI 2005, pp. 191-200. ACM Press, New York (2005)

12. Walther, J.B.: Computer-mediated communication: Impersonal, interpersonal, and hyperpersonal interaction. Communication Research 23, 3-43 (1996) 